\title{
Aging and the discrimination of 3-D shape from motion and binocular disparity
}

\author{
J. Farley Norman • Jessica S. Holmin • \\ Amanda M. Beers • Jacob R. Cheeseman • \\ Cecilia Ronning • Angela G. Stethen • Adam L. Frost
}

Published online: 6 July 2012

(C) Psychonomic Society, Inc. 2012

\begin{abstract}
Two experiments evaluated the ability of younger and older adults to visually discriminate $3-\mathrm{D}$ shape as a function of surface coherence. The coherence was manipulated by embedding the 3-D surfaces in volumetric noise (e.g., for a $55 \%$ coherent surface, $55 \%$ of the stimulus points fell on a 3-D surface, while $45 \%$ of the points occupied random locations within the same volume of space). The 3-D surfaces were defined by static binocular disparity, dynamic binocular disparity, and motion. The results of both experiments demonstrated significant effects of age: Older adults required more coherence (tolerated volumetric noise less) for reliable shape discrimination than did younger adults. Motion-defined and static-binoculardisparity-defined surfaces resulted in similar coherence thresholds. However, performance for dynamic-binoculardisparity-defined surfaces was superior (i.e., the observers' surface coherence thresholds were lowest for these stimuli). The results of both experiments showed that younger and older adults possess considerable tolerance to the disrupting effects of volumetric noise; the observers could reliably discriminate 3-D surface shape even when $45 \%$ of the stimulus points (or more) constituted noise.
\end{abstract}

Keywords Binocular vision: stereopsis $\cdot 3 \mathrm{D}$ perception: depth and shape from $X \cdot$ Aging

\footnotetext{
J. F. Norman

Department of Psychology \& Center for the Study of Lifespan Development, Western Kentucky University,

Bowling Green, KY 42101-1030, USA

J. F. Norman $(\varangle) \cdot$ J. S. Holmin • A. M. Beers • J. R. Cheeseman C. Ronning $\cdot$ A. G. Stethen $\cdot$ A. L. Frost

Department of Psychology, Western Kentucky University,

1906 College Heights Blvd. \#21030,

Bowling Green, KY 42101-1030, USA

e-mail: Farley.Norman@wku.edu
}

\section{Aging and the discrimination of 3-D shape from motion and binocular disparity}

The informativeness of binocular disparity and motion parallax for the perception of depth and solid object shape has been known and discussed since the 19th century (e.g., Helmholtz, 1867/1925; Ogle, 1950, 1958; Wheatstone, 1838). While early investigators employed a variety of surface shapes defined by binocular disparity and/or motion (e.g., Braunstein, 1966; Green, 1961; Julesz, 1971; Johansson, 1975; Ullman, 1979; Wallach \& O'Connell, 1953), vision researchers did not actually measure human observers' ability to discriminate 3-D surface shape until the 1980s and 1990s (e.g., de Vries, Kappers, \& Koenderink, 1993; Norman \& Lappin, 1992; Norman, Lappin, \& Zucker, 1991; Rogers \& Graham, 1979; Sperling, Landy, Dosher, \& Perkins, 1989; Uttal, Davis, Welke, \& Kakarala, 1988; Van Damme \& Van de Grind, 1993). Such psychophysical research into shape discrimination has continued to the present day (e.g., Norman, Beers, Holmin, \& Boswell, 2010; Norman, Swindle, Jennings, Mullins, \& Beers, 2009; Vreven, 2006).

One important fact about human vision is that observers can effectively perceive continuous 3-D surfaces even when random-dot stereograms or kinetic depth effect displays possess very low density (i.e., when the 3 -D surfaces are sparsely sampled). For example, Norman and Lappin (1992, Fig. 5) showed that observers can effectively perceive and discriminate 3-D surface shape even when surfaces are defined only by the motions and 3-D positions of nine points (see also Julesz, 1971; Lappin \& Craft, 2000; Norman, Dawson, \& Butler, 2000; Uttal et al., 1988). To achieve the perception of whole surfaces from a sparse sampling of motion and/or binocular disparity requires interpolation or approximation (see, e.g., Dinh, Turk, \& Slabaugh, 2002; Howard \& Rogers, 2012, pp. 474-477; 
Marr, 1982, pp. 285-287, 290-291; Saidpour, Braunstein, \& Hoffman, 1994; Terzopoulos, 1988; Vuong, Domini, \& Caudek, 2004; Wilcox \& Duke, 2005; Yang \& Blake, 1995), where information about surface shape and depth is propagated from surface regions possessing well-defined depths into regions without depth. Consider Fig. 1, which depicts a stereoscopic surface similar to those used in the present investigation. Algorithms, such as those developed by Terzopoulos, can reconstruct an entire 3-D surface from sparse image data - in part, by assuming that an object's constituent depths and surface orientations typically vary in a relatively smooth manner across its surface.

We know from the previous research cited above that 3-D surface interpolation is an important visual process. What we do not know well at the moment are the limits to human surface interpolation. Consider Fig. 2. The upper-left panel depicts a sparsely sampled 3-D surface; in this example, the best interpolation is straightforward (i.e., the solid curve in between the well-defined surface points). However, the lower-left panel depicts a "noisy" version of the same $3-\mathrm{D}$ surface $(60 \%$ of the points occupy depths that are identical to those of the original surface, while $40 \%$ of the original surface points have been relocated to random positions; in our terminology, this surface has $60 \%$ coherence). How should interpolation proceed in this situation, where there is a coherent 3-D surface embedded in "volumetric noise"? Note from the lower-left panel that, if all of the points are treated as equivalent, a reasonable interpolation (solid curve) bears no resemblance to the original 3-D surface. Now consider the lower-right panel. If human vision is particularly sensitive to smooth surfaces and local violations of that smoothness, the "noise points" can be identified and segregated, with the interpolation (solid curve) being applied only to the remaining "surface points." It is thus possible that human vision could be remarkably tolerant to 3-D surfaces that are embedded in noise.

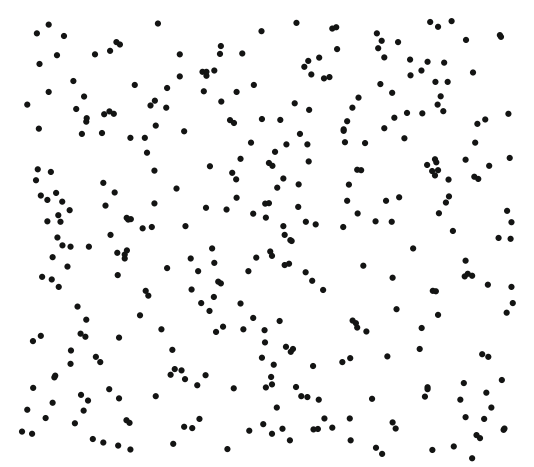

Fig. 1 Random-dot stereogram depicting a 3-D curved surface (a "Bulls-Eye") similar to the stimuli used in the present experiments. This example surface is fully "coherent" (i.e., no volumetric noise is present). This stereogram was designed for crossed free-fusion but will
To what extent can human observers perceive and discriminate 3-D surface shapes that are embedded in volumetric noise? Consider the stereogram presented in Fig. 3, with $30 \%$ noise points occupying random depths within the surface's overall volume (i.e., the surface is $70 \%$ coherent); it is clearly more difficult (than in Fig. 1) to extract and perceive the embedded surface shape, despite the fact that more than two thirds of the points within the stereogram are located on a coherent 3-D surface. How much volumetric noise can human observers tolerate when discriminating 3D shape? There are surprisingly few answers to this question. Uttal (1985) investigated the detection (i.e., presence or absence) of curved surfaces embedded in volumetric noise but did not address shape discrimination. Uttal et al. (1988) did investigate stereoscopic shape discrimination but did not utilize or manipulate volumetric noise. In contrast, Norman et al. (1991) required observers to discriminate the stereoscopic shape of surfaces that were embedded in various amounts of volumetric noise. In particular, their observers discriminated between triangle waves modulated in depth (which therefore possessed discontinuities in surface orientation) and sinusoidal surfaces modulated in depth (which were smoothly curved and did not possess discontinuities). Norman et al. (1991) found that as long as the spatial frequency of the depth modulations was 1 cycle/degree (visual angle) or below, their observers could reliably perform 3-D shape discrimination at above-chance levels even when $30 \%$ of the points in the random-dot stereograms constituted noise (i.e., $70 \%$ of the 640 points defined the surfaces, while the remaining $30 \%$ of the points occupied random depths within the same volume of space).

Interpolation helps observers to perceive coherent 3-D surfaces when sparse optical information about 3-D structure is provided from either binocular disparity or motion (e.g., Saidpour et al., 1994; Terzopoulos, 1988; Vuong, Domini, \& Caudek, 2006; Wilcox \& Duke, 2005). The presence of volumetric noise, as was discussed earlier,

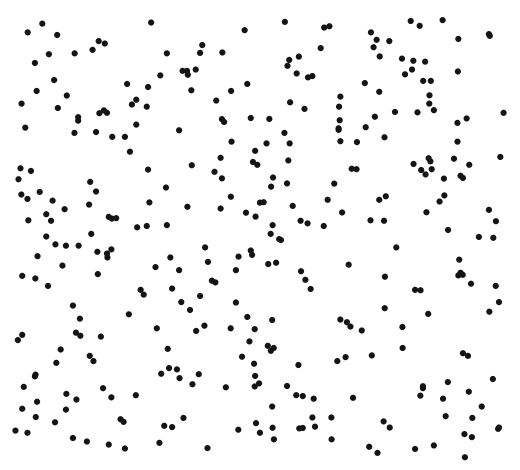

work just as well with divergent free-fusion (the depth relief will be reversed). Alternately, the two half-images of the stereogram may be viewed in a stereoscope 
$100 \%$ coherence

interpolate surface between points

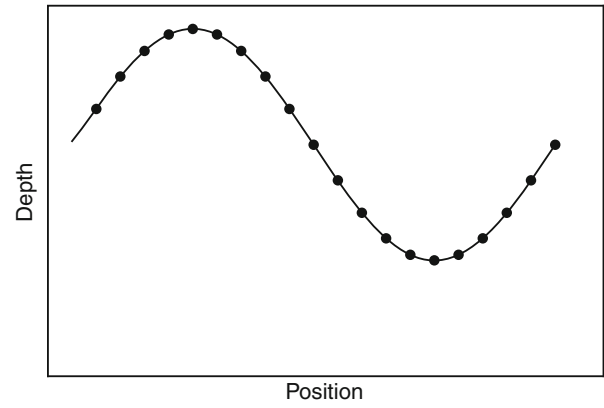

$60 \%$ coherence $40 \%$ volumetric noise

interpolate smooth surface between points

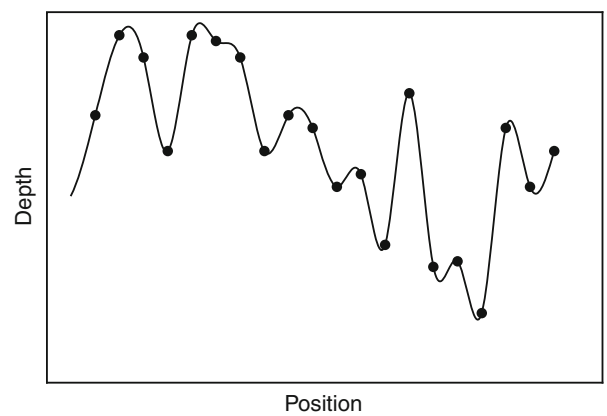

Fig. 2 The upper-left panel depicts a cross section of a sparsely defined and smoothly curved surface modulated in depth. The solid curve indicates a reasonable interpolation (i.e., a smooth continuous surface consistent with the given points). The bottom-left panel shows the same surface, except that, in this case, only $60 \%$ of the previous points fall on the smoothly curved surface. The other $40 \%$ of the points represent "noise" and occupy random locations within the same volume of space. If all of the given points are treated equivalently and a smooth surface is interpolated between them, one obtains the resulting solid curve. Note that the solid curve in this case bears no resemblance

would be expected to impair the perception of 3-D surfaces and, consequently, limit the ability of human observers to discriminate 3-D shape. Three-dimensional shape perception and discrimination is important throughout the lifespan: Older human observers depend upon 3-D shape perception as much as younger observers while performing their everyday activities. While the ability to discriminate 3-D shape has been assessed in older adults (60-84 years of age) for
$60 \%$ coherence $40 \%$ volumetric noise identify and discount noise points, then interpolate surface

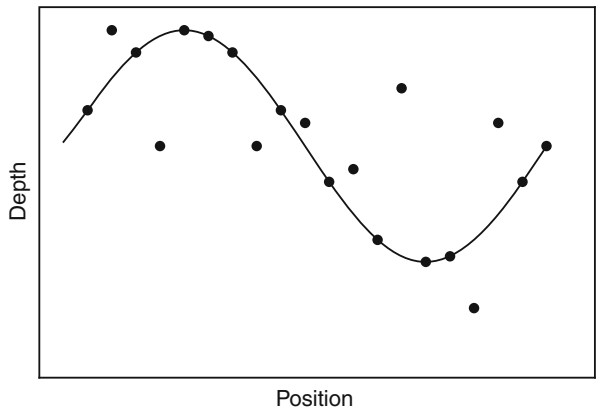

to the original surface. The bottom-right panel depicts the same points as in the bottom-left panel (i.e., $60 \%$ of the points fall on a smoothly curved surface, and the surface is obscured by the remaining volumetric noise points). If human vision is sensitive to the smoothness of 3-D surfaces and violations of that smoothness, the noise points can potentially be identified and segregated. If the resulting interpolation is then applied to only the surface points (because the noise points have been segregated), one obtains a smooth interpolated surface identical to that obtained in the top-left panel

surfaces defined by both motion and binocular disparity (e.g., Norman, Clayton, Shular \& Thompson 2004; Norman, Crabtree, Norman, et al., 2006; Norman et al., 2000), no psychophysical study has ever investigated aging and surface interpolation. Does volumetric noise affect 3-D shape discrimination similarly in younger and older observers? If not, does volumetric noise impair shape discrimination more severely in older observers? The answers to such
Fig. 3 Random-dot stereogram depicting a "Bulls-Eye" surface embedded in volumetric noise. In this example, $70 \%$ of the disparate points fall on the curved surface, while $30 \%$ of the points are randomly placed throughout the same volume of space
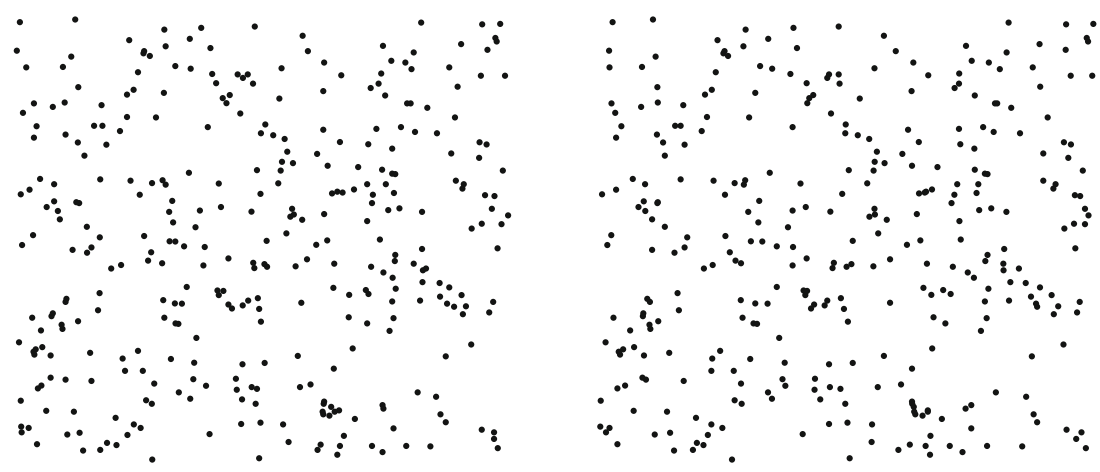
questions are entirely unknown. From our laboratory's prior research, we know that older observers can discriminate solid 3-D shape just as well as younger observers under favorable circumstances (see Fig. 2 of Norman, Crabtree, Norman, et al., 2006). We also know, however, that significant age differences emerge in the discrimination of 3-D shape from motion when the temporal correspondence of surface points is disrupted (Norman et al., 2004). Given these previous results, it is likely that while older adults may discriminate 3-D shape well when surface coherence is high, their performance will deteriorate (more than that of younger observers) when surface coherence is low. The present study is the first to compare 3-D shape discrimination abilities in younger and older adults when surface interpolation processes are disrupted by large amounts of volumetric noise.

\section{Experiment 1: Stereoscopic shape discrimination}

\section{Method}

Apparatus Stereograms were created by an Apple PowerMacintosh G4 computer and displayed on a 22 -in. Mitsubishi Diamond Plus 200 color monitor (resolution was $1,280 \times 1,024$ pixels). The viewing distance was $100 \mathrm{~cm}$.

Experimental stimuli The experimental stimuli were static and dynamic random-dot stereograms (the projected width and height of the stereograms was $21.5^{\circ}$ and $14.5^{\circ}$ of visual angle, respectively); they were presented as anaglyphs (see, e.g., Fox, Aslin, Shea, \& Dumais, 1980; Julesz, 1971; Norman, Burton, \& Best, 2010; Norman, Crabtree, Herrmann, et al., 2006; Patterson et al., 1995; Patterson, Moe, \& Hewitt, 1992). The stereograms contained either 1,000 or 6,000 binocularly disparate points; these disparities defined both the experimental surfaces and the volumetric noise. The depicted surfaces were smoothly curved in depth and were similar to those used by Norman et al. (2004). In particular, there were three types of surfaces, whose variations in depth were sinusoidal. In one surface type (the "Bulls-Eye," shown in Fig. 1), the depth was modulated sinusoidally as a function of increasing distance from the center of a stereogram, resulting in a series of circular peaks and troughs. A second surface type (the "Star" or "Snowflake") was characterized by sinusoidal peaks and troughs that radiated outward from the center of a stereogram (if the point positions within a stereogram are defined in terms of polar coordinates, $r$ and $\theta$, then the depth of these surfaces was modulated sinusoidally as a function of $\theta$ ). In the final surface type (the "Egg-crate"), the depth was modulated sinusoidally as functions of both $x$ - and $y$-coordinates within a stereogram [i.e., $z=\sin (x) * \sin (y)$ ]; these surfaces possessed arrays of "bumps" and "dimples."
The average spatial frequency for all surfaces was 0.3 cycles/degree of visual angle, which is near the peak of the stereoscopic modulation transfer function (see, e.g., Fig. 4 of Rogers \& Graham, 1982). The phases of these sinusoidal depth modulations were randomly determined for each trial, so that each experimental stimulus was unique. The maximum image disparity within each stereogram was $0.3 \mathrm{~cm}$ (crossed and uncrossed). For an observer with an interpupillary distance of $6.1 \mathrm{~cm}$, these image disparities produce front-to-back (i.e., peak-totrough) perceived depths of $11.6 \mathrm{~cm}$ (Cormack \& Fox, 1985). The corresponding amount of binocular disparity (between the front and back of the stereoscopic surfaces) was 24.6 minutes arc.

Procedure There were a total of 15 experimental conditions (14 low-density conditions with 1,000 points per stereogram and 1 high-density no-noise "control" condition with 6,000 points per stereogram). The 14 lowdensity conditions were derived from the orthogonal combination of two stereogram types (static and dynamic stereograms) and seven values of surface "coherence" $(100 \%, 85 \%, 70 \%, 55 \%, 40 \%, 25 \%$, and $10 \%$; see Norman et al., 1991). A coherence value of $70 \%$, for example, indicates that $70 \%$ of a stereogram's points defined a smoothly curved surface in 3-D space, while $30 \%$ of the points constituted volumetric "noise" (i.e., points occupying random depths within the same volume of space as the depicted 3-D surface).

On each individual trial, an observer would be presented with either a static (i.e., unchanging) or a dynamic random-dot stereogram for $5 \mathrm{~s}$. In the dynamic case, a completely new stereogram was presented every $28.6 \mathrm{~ms}$; even though the disparate points appeared (and disappeared) at a rate of $35 \mathrm{~Hz}$, the underlying or "implicit" surface remained the same throughout the trial. Because an entirely new set of disparate points was presented every $28.6 \mathrm{~ms}$, there was no coherent motion at all; the apparent motion signals were random and uncorrelated (our dynamic stereograms were similar to Julesz's [1971] "Stereo Movie 2," which is described in his section 5.10, pp. 183-184). The observers' task on each trial was to indicate which 3-D surface had been presented: "Bulls-Eye," "Star," or "Egg-crate."

Within each experimental session, the observers judged 90 trials $(15$ conditions $\times 3$ surface shapes [Bulls-Eye, Star, and Egg-crate] $\times 2$ replications). The order of the conditions and 3-D shapes within a session was completely random. Each observer participated in five experimental sessions. All sessions for each observer were run on the same day, with short breaks between sessions. By the end of the experiment, each observer had judged a total of 450 stereograms (5 sessions $\times 90$ trials/session). 
Fig. 4 Experimental results: Individual results for representative younger and older observers in Experiment 1. The observers' shape discrimination accuracies are plotted as a function of surface coherence. The percentage of volumetric noise for each condition equals 100 minus the surface coherence values (e.g., $45 \%$ of the points in stereograms that depict surfaces with $55 \%$ coherence occupy random depths within the same volume of space). The bestfitting psychometric functions (i.e., Weibull fits) are illustrated by the solid curves. The dashed horizontal lines indicate chance levels of performance. The left column shows the observers' results for static stereograms, while the right column indicates results for dynamic stereograms
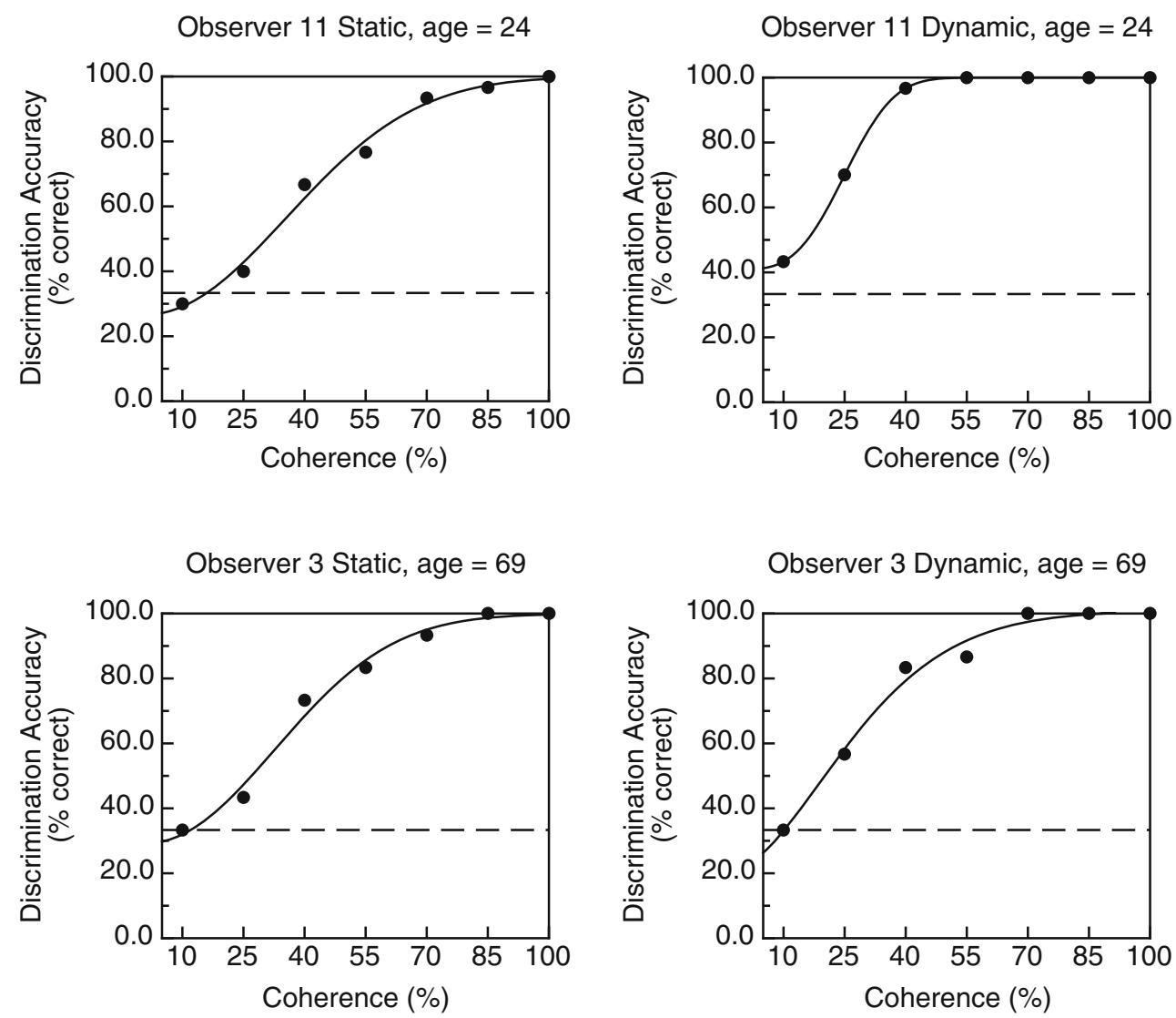

Observers Twenty-one observers participated in the experiment. One group of observers consisted of 11 older adults (mean age was 70.7 years, $S D=2.6$; the range of their ages was $65-75$ years). The other group consisted of 10 younger adults (mean age was 23.1 years, $S D=$ 3.3 ; range of ages was $18-28$ years). The observers' acuities were assessed with a standard ETDRS eye chart (Precision Vision catalog number 2195) at a distance of $1 \mathrm{~m}$. Both the younger and older observers possessed good visual acuity: The younger observers' average acuity was -0.14 LogMAR (log minimum angle of resolution), while the older observers' average acuity was $-0.01 \operatorname{LogMAR}$ (a zero value of LogMAR indicates "normal" acuity, while negative and positive values indicate "better than average" and "less than average" acuity, respectively). All 21 observers were naïve and had no knowledge of the previous literature, exact hypotheses under test, and so forth.

Results and discussion

The older and younger observers' stereoscopic shape discrimination performances for the static-stereogram, high-density, no-noise "control" condition were $99.1 \%$ and $100 \%$ correct, respectively. It is obvious that this difference in performance is neither significant, $t(10.0)=$
$1.4, p=.19$, nor of practical importance. Both age groups perform well when the surface point density is high and there is no volumetric noise.

Representative results for individual younger and older observers are illustrated in Fig. 4; these graphs plot the observers' shape discrimination performance as a function of surface coherence (e.g., $70 \%$ coherence indicates that $70 \%$ of stimulus points were located on an implicit 3-D surface, while the remaining $30 \%$ of points occupied random locations within the same volume of space; see Norman et al., 1991). For all of the observers in both the static and dynamic stereogram conditions, we fit a Weibull function to their data (Macmillan \& Creelman, 1991, p. 190). These Weibull fits (i.e., psychometric functions) are indicated by the solid curves in Fig. 4. From these psychometric functions, the observers' surface coherence thresholds were estimated; in particular, the surface coherence that resulted in $66.7 \%$ correct discrimination accuracy (where performance was halfway between chance and perfect) was used as the observers' threshold. The surface coherence thresholds for all observers are plotted in Fig. 5. As is readily evident, dynamic stereograms led to superior performance (i.e., lower thresholds), $F(1,19)=37.4, p<.00001, \eta_{\mathrm{p}}{ }^{2}=.66$. In addition, there was a main effect of age, $F(1,19)=7.4, p<.02$, 


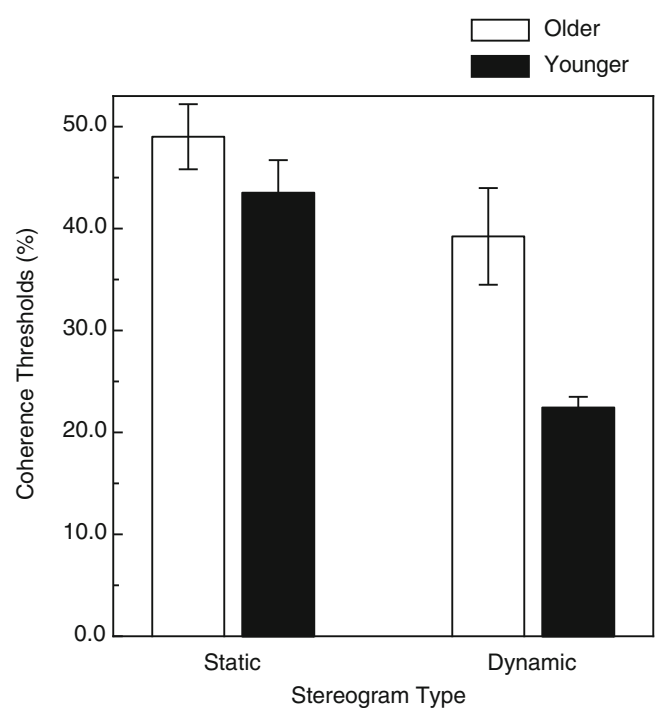

Fig. 5 Experimental results: Overall results (coherence thresholds) obtained for younger and older observers in Experiment 1. The thresholds are plotted separately for static and dynamic random-dot stereograms. The error bars indicate $\pm 1 S E$

$\eta_{\mathrm{p}}{ }^{2}=.28$. Finally, there was a significant age $\times$ stereogram type interaction, $F(1,19)=5.0, p<.04, \eta_{\mathrm{p}}{ }^{2}=$ .21. The younger observers' thresholds were $11.2 \%$ lower than the older observers' thresholds for the static stereograms but were $42.8 \%$ lower when the 3-D surfaces were defined by dynamic stereograms. Thus, one can see that while there was an overall deterioration in performance associated with age, the largest deterioration occurred in the dynamic conditions.

\section{Experiment 2: Discrimination of 3-D shape from motion}

Experiment 1 was designed to evaluate observers' ability to stereoscopically discriminate 3-D shape as a function of surface coherence. The results demonstrated that while there are significant age-related differences in performance, human observers' shape discrimination ability is tolerant of large disruptions in surface coherence: in particular, both older and younger observers could reliably discriminate surface shape when fewer than half of the stimulus points fell on coherent surfaces (and when the majority of the stimulus points thus served as masking "volumetric noise"). Because human observers effectively perceive 3-D shape from motion in addition to binocular disparity (e.g., Andersen, 1996; Braunstein, 1966; Domini, Caudek, \& Richman, 1998; Norman \& Lappin, 1992; Todd, Akerstrom, Reichel, \& Hayes, 1988), Experiment 2 was designed to similarly evaluate the effects of reductions in surface coherence for 3-D surfaces defined by motion.
Method

Apparatus The apparatus was identical to that used in Experiment 1. The viewing distance was again $100 \mathrm{~cm}$. The observers viewed the stimuli monocularly through a viewing hood (Norman, Beers, et al., 2010; Todd \& Norman, 1991).

Experimental stimuli The depicted surfaces were identical to the low-density surfaces (i.e., 1,000 points) used in Experiment 1 in terms of both shape ("Bulls-Eye," "Star,", and "Egg-crate") and front-to-back depth, except that the 3-D surfaces were now defined by motion. The surfaces oscillated (i.e., rotated) in depth about a Cartesian vertical axis located in the plane of the computer monitor. The maximum extent of the surface rotation from a "frontal" orientation was $\pm 5^{\circ}$. In order to prevent abrupt changes, the surfaces decelerated before changing rotation direction; the average rotation increment was $0.23^{\circ}$ per frame transition. The individual frames were updated at a rate of $35 \mathrm{~Hz}$.

Procedure As in Experiment 1, there were a total of seven coherence conditions (surface coherences of $100 \%, 85 \%, 70 \%, 55 \%, 40 \%, 25 \%$, and $10 \%$ ). Once again, the "noise" points in the various coherence conditions were randomly positioned within the same volume of space occupied by the depicted 3-D surfaces. The observers' task on each trial was identical to that employed in Experiment 1: Observers were required to indicate which of the three surfaces had been presented ("Bulls-Eye," "Star," or "Egg-crate").

Within each experimental session, the observers judged 84 trials $(7$ conditions $\times 3$ surface shapes [Bulls-Eye, Star, and Egg-crate] $\times 4$ replications). The order of the coherence conditions and 3-D shapes within a session was completely random. Each observer participated in four experimental sessions, all conducted on the same day. Therefore, by the end of the experiment, each observer had judged a total of 336 stimulus presentations.

Observers Sixteen observers participated in the experiment. One group of observers consisted of 8 older adults (mean age was 73.8 years, $S D=6.3$; the range of their ages was $67-85$ years). The other group consisted of 8 younger adults (mean age was 22.3 years, $S D=3.4$; range of ages was 18-28 years). One potential younger observer was excluded because she did not understand the task. The observers' acuities were assessed with a standard ETDRS eye chart (Precision Vision catalog number 2195) at a distance of $1 \mathrm{~m}$. Both the younger and older observers possessed good visual acuity: The younger observers' average acuity was $-0.125 \operatorname{LogMAR}$, 
while the older observers' average acuity was 0.025 LogMAR. Two of the younger observers were coauthors, while the remaining 14 participants were naïve. None of the observers in the present experiment had participated in Experiment 1.

\section{Results and discussion}

Representative results for individual younger and older observers are depicted in Fig. 6; the observers' shape discrimination performance is plotted as a function of surface coherence (e.g., $55 \%$ coherence indicates that $55 \%$ of stimulus points were located on an implicit 3-D surface, while the remaining $45 \%$ of points occupied random locations within the same volume of space). To determine the observers' coherence thresholds, we again fit a Weibull function (shown by the solid curves in Fig. 6) to their data. The surface coherence thresholds for the present experiment are indicated by the black bars in Fig. 7. One can readily see that there was a significant effect of age in the present experiment, $t(14)=-3.3, p=.005$, two-tailed: The older observers' coherence thresholds for motion-defined surfaces were $50 \%$ higher than those obtained for the younger observers. The shape discrimination performance obtained in the present experiment involving motion can be compared with the analogous performance obtained for the static (white bars) and dynamic (crosshatched bars) binocular disparity conditions of Experiment 1. A two-way between-subjects analysis of variance comparing the present motion results with the static disparity results of Experiment 1 revealed that there was a consistent effect of age in both experiments, $F(1,33)=11.4$, $p=.002, \eta_{\mathrm{p}}{ }^{2}=.26$. Overall, there was no difference between the coherence thresholds obtained in the static disparity condition of Experiment 1 and those of the present experiment, $F(1,33)=$ $0.01, p=.92, \eta_{\mathrm{p}}{ }^{2}<.001$. Although the present thresholds obtained for motion-defined surfaces were slightly higher for the older observers (relative to the static disparity condition of Experiment 1) and were slightly lower for the younger observers, the interaction between age and optical information (binocular disparity vs. motion) was not significant, $F(1,33)=3.3, p=.08$.
The pattern of results is different, however, if one compares the thresholds obtained for the motion-defined surfaces of the present experiment with those of the analogous dynamic binocular disparity condition used in Experiment 1. In this case, not only was there a main effect of age, $F(1,33)=21.6, p<.001, \eta_{\mathrm{p}}{ }^{2}=$ .40 , but also there was a significant effect of optical information (i.e., dynamic binocular disparity vs. motion), $F(1,33)=15.9, p<.001, \eta_{\mathrm{p}}{ }^{2}=.33$. The coherence thresholds for surfaces defined by dynamic binocular disparity were $33 \%$ lower than those for surfaces defined by motion [the interaction between type of optical information and age was once again not significant, $F(1,33)=0.04, p=.84]$.

\section{General discussion}

In 1991, Norman et al. demonstrated that human stereopsis is sensitive to the smoothness of 3-D surfaces. Small deviations in coherence (e.g., $4 \%-10 \%$ ) from fully coherent surfaces were easily detected by human observers, while those same differences were much less discriminable from less coherent surfaces. In their third experiment, Norman et al. (1991) required observers to discriminate between a smooth sinusoidal surface modulated in depth and a triangle wave surface (characterized by abrupt, nonsmooth, changes in surface orientation - i.e., sharp corners) of the same spatial frequency. Their Fig. 5 (p. 800) shows that as long as the spatial frequency was 1 cycle/deg or less, the observers were sensitive to and could discriminate between these smooth and nonsmooth 3-D surfaces even when they were embedded within large amounts (30\%) of volumetric noise. More recently, Vreven (2006; see her Figs. 3 and 6) demonstrated that stereoscopic surfaces whose shapes differed in smoothness (e.g., a "pointy" surface with a sudden change in orientation at an apex vs. a curved surface characterized by gradual changes in orientation) are especially discriminable, as compared with surfaces that do not qualitatively differ in smoothness.
Fig. 6 Experimental results: Individual results for representative younger and older observers in Experiment 2. The observers' shape discrimination accuracies are plotted as a function of surface coherence. The best-fitting psychometric functions (i.e., Weibull fits) are illustrated by the solid curves. The dashed horizontal lines indicate chance levels of performance
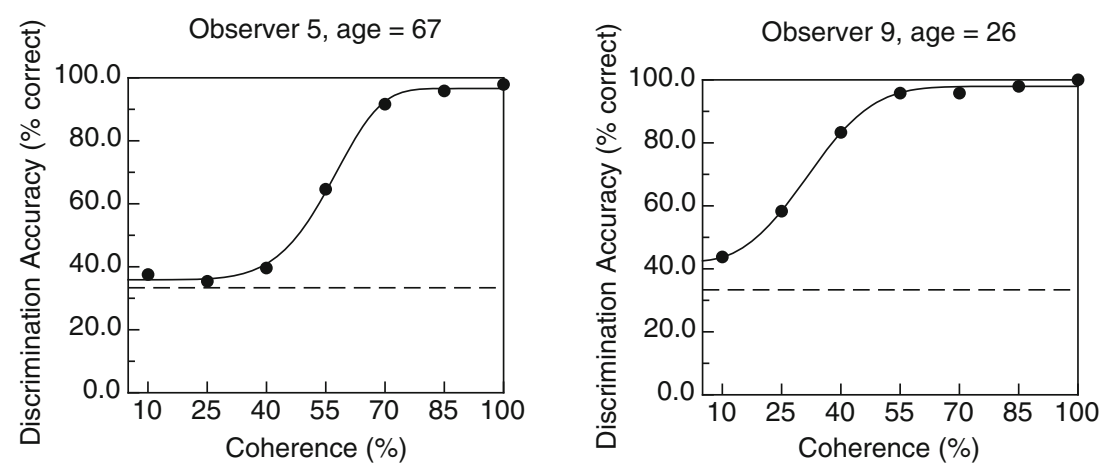


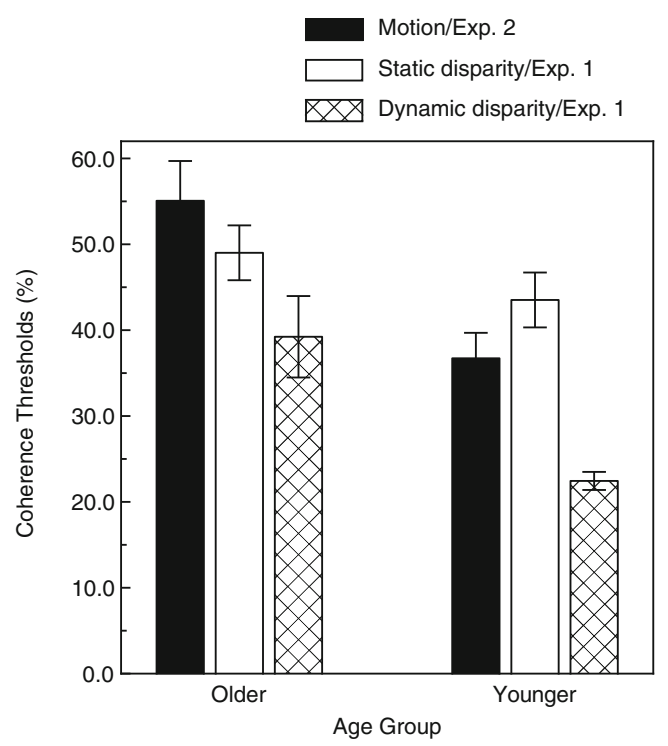

Fig. 7 Results for Experiment 1 and 2: Overall results (coherence thresholds) obtained for younger and older observers. The results of Experiment 2 for motion-defined surfaces are indicated by the black bars, while the analogous results of Experiment 1 for surfaces defined by static and dynamic binocular disparity are indicated by the white and crosshatched bars, respectively. The error bars indicate $\pm 1 S E$

If human stereopsis is sensitive to surface smoothness, as the results of previous psychophysical studies indicate (see also Marr \& Poggio, 1976), and local violations of smoothness are therefore salient and identifiable, 3-D shape discrimination should be tolerant of large amounts of volumetric noise (see Fig. 2 in the present article). The results of our present Experiment 1 demonstrate that human stereopsis is indeed tolerant of the disrupting effects of volumetric noise; all of our observers, whether younger or older, could reliably discriminate 3-D shape when most of the stimulus points constituted "noise" (and thus, when only a minority of the stimulus points fell on the depicted 3-D surfaces themselves). The present results thus extend and reinforce the earlier findings of Norman et al. (1991). The present investigation also extends the research of Norman et al. (1991) in another important direction, to the perception and discrimination of 3-D shape from motion. The results of the present Experiment 2 (see Fig. 7) demonstrate that volumetric noise affects the discrimination of 3-D shape from motion in a manner that is similar to how it affects the discrimination of 3D shape from static binocular disparity. It does not appear to matter whether 3-D shape is specified by motion or static binocular disparity; 3-D surface interpolation and the resulting shape discrimination proceed similarly.

The present Experiment 1 replicates and extends a phenomenon observed by Norman, Crabtree, Herrmann, et al.
(2006); they found that younger observers' 3-D shape discrimination performance was facilitated by dynamic binocular disparity (when compared with analogous conditions where the 3-D surfaces were defined by static binocular disparity-i.e., conventional stereograms). In these dynamic stereograms, a single 3-D shape was presented throughout any given trial; however, the disparate points that defined the shape were replaced (completely changed) every 28.6 or $14.3 \mathrm{~ms}$ (replaced at 35 or $70 \mathrm{~Hz}$ ). Even though the specified surfaces did not change throughout a trial, the increased spatial sampling of the underlying "implicit" surfaces that occurred over time led to improved shape discrimination performance. This improvement accompanying the usage of dynamic random-dot stereograms also occurred in the present investigation (see Figs. 5 and 7): In particular, the older observers' shape discrimination performance improved by $20 \%$, while the younger observers' performance improved by $48.4 \%$. While it has been known for decades (e.g., Fox et al., 1980; Julesz, 1971) that people can perceive 3-D shape from dynamic random-dot stereograms, our laboratory's current and recent research demonstrates that dynamic stereograms facilitate human shape discrimination performance above that obtained with conventional stereograms and conventional structure-from-motion (i.e., kinetic depth effect) displays.

Past research has demonstrated that there is no overall age-related impairment for the visual perception and discrimination of 3-D shape. For example, for a 3-D same/ different shape discrimination task where the experimental stimuli were naturally shaped objects (bell peppers) presented with motion, binocular disparity, shading, and specular highlights, Norman, Crabtree, Norman, et al. (2006) found that older and younger adults performed at identical levels of accuracy $(83.9 \%$ and $83.8 \%$ correct for older and younger adults, respectively). Despite this good performance for older adults under full-cue conditions, their shape discrimination performance frequently deteriorates when conditions are less favorable - for example, (1) when binocular correspondence is reduced (Norman, Crabtree, Hermann, et al., 2006), (2) when the lifetimes of individual surface points are reduced in a perceived structure-frommotion task (Norman et al., 2004; Norman et al., 2000), and (3) when high magnitudes of binocular disparity are present (Norman et al., 2008). The results of the present set of experiments are consistent with this overall pattern. In the present experiments, where the 3-D surfaces were defined by either binocular disparity (Experiment 1 ) or motion (Experiment 2), the observers were required to discriminate the shapes of 3-D surfaces that were highly degraded by volumetric noise. Consistent with our past findings, an agerelated deficit in performance was obtained in both experiments (because the severe reductions in coherence disrupted the interpolation processes needed to perceive and discriminate surface shape). 
It is interesting that the magnitude of the age deficit in the present investigation was smallest when the 3-D surfaces were defined by static binocular disparity and was largest when the 3-D surfaces were defined by motion and dynamic binocular disparity. Older adults often have difficulty performing any task involving motion, even if it does not concern shape. For example, older adults' ability to discriminate the speed of moving patterns is much worse than younger adults' (e.g., Norman, Burton, \& Best, 2010; Norman, Ross, Hawkes, \& Long, 2003; Raghuram, Lakshminarayanan, \& Khanna, 2005; Snowden \& Kavanagh, 2006). Our present finding that the age-related deficit for discriminations of 3-D shape is higher for motion than for static binocular disparity is consistent with the idea that aging negatively affects performance on most, if not all, psychophysical tasks involving motion (see also Atchley \& Andersen, 1998; Bennett, Sekuler, \& Sekuler, 2007; Betts, Taylor, Sekuler, \& Bennett, 2005). This age-related deficit for motion tasks has recently been associated with reductions in GABA (gammaaminobutyric acid) activity in cortical area MT and the primary visual cortex (Leventhal, Wang, $\mathrm{Pu}$, Zhou, \& Ma, 2003; Liang et al., 2010; Yang, Liang, Li, Wang, \& Zhou, 2009; Yang, Zhang, et al., 2009). Thus, it is likely that at least some of the age-related deficits observed in the present experiments were caused by deterioration in the functionality of inhibitory mechanisms within the primary visual cortex and/or cortical area MT. Since Leventhal et al. were able to restore normal neuronal functionality in old monkeys by applying GABA (or muscimol, a GABA agonist) directly to the primary visual cortex, it may be possible to develop effective treatments in the future that improve older adults' abilities to utilize motion for the perception of 3-D shape.

Author Notes The authors would like to thank three anonymous reviewers for their constructive comments and helpful suggestions during the preparation of the manuscript. The reviewers' suggestions led directly to the development and inclusion of Experiment 2 into the present study.

\section{References}

Andersen, G. J. (1996). Detection of smooth three-dimensional surfaces from optic flow. Journal of Experimental Psychology. Human Perception and Performance, 22, 945-957.

Atchley, P., \& Andersen, G. J. (1998). The effect of age, retinal eccentricity, and speed on the detection of optic flow components. Psychology and Aging, 13, 297-308.

Bennett, P. J., Sekuler, R., \& Sekuler, A. B. (2007). The effects of aging on motion detection and direction identification. Vision Research, 47, 799-809.

Betts, L. R., Taylor, C. P., Sekuler, A. B., \& Bennett, P. J. (2005). Aging reduces center-surround antagonism in visual motion processing. Neuron, 45, 361-366.
Braunstein, M. L. (1966). Sensitivity of the observer to transformations of the visual field. Journal of Experimental Psychology, 72, 683-689.

Cormack, R., \& Fox, R. (1985). The computation of disparity and depth in stereograms. Perception \& Psychophysics, 38, 375-380.

de Vries, S. C., Kappers, A. M., \& Koenderink, J. J. (1993). Shape from stereo: A systematic approach using quadratic surfaces. Perception \& Psychophysics, 53, 71-80.

Dinh, H. Q., Turk, G., \& Slabaugh, G. (2002). Reconstructing surfaces by volumetric regularization using radial basis functions. IEEE Transactions on Pattern Analysis and Machine Intelligence, 24, $1358-1371$.

Domini, F., Caudek, C., \& Richman, S. (1998). Distortions of depthorder relations and parallelism in structure from motion. Perception \& Psychophysics, 60, 1164-1174.

Fox, R., Aslin, R. N., Shea, S. L., \& Dumais, S. T. (1980). Stereopsis in human infants. Science, 207, 323-324.

Green, B. F. (1961). Figure coherence in the kinetic depth effect. Journal of Experimental Psychology, 62, 272-282.

Howard, I. P., \& Rogers, B. J. (2012). Perceiving in depth (Vol. 2, Stereoscopic vision). New York: Oxford University Press.

Johansson, G. (1975). Visual motion perception. Scientific American, 232(6), 76-88

Julesz, B. (1971). Foundations of cyclopean perception. Chicago: University of Chicago Press.

Lappin, J. S., \& Craft, W. D. (2000). Foundations of spatial vision: From retinal images to perceived shapes. Psychological Review, 107, 6-38.

Leventhal, A. G., Wang, Y., Pu, M., Zhou, Y., \& Ma, Y. (2003). GABA and its agonists improved visual cortical function in senescent monkeys. Science, 300, 812-815.

Liang, Z., Yang, Y., Li, G., Zhang, J., Wang, Y., Zhou, Y., \& Leventhal, A. G. (2010). Aging affects the direction selectivity of MT cells in rhesus monkeys. Neurobiology of Aging, 31, 863-873.

Macmillan, N. A., \& Creelman, C. D. (1991). Detection theory: A user's guide. New York: Cambridge University Press.

Marr, D. (1982). Vision: A computational investigation into the human representation and processing of visual information. San Francisco: Freeman.

Marr, D., \& Poggio, T. (1976). Cooperative computation of stereo disparity. Science, 194, 283-287.

Norman, J. F., Beers, A. M., Holmin, J. S., \& Boswell, A. M. (2010). Effective 3-D shape discrimination survives retinal blur. Attention, Perception, \& Psychophysics, 72, 1569-1575. doi:10.3758/ APP.72.6.1569

Norman, J. F., Burton, C. L., \& Best, L. A. (2010). Modulatory effects of binocular disparity and aging upon the perception of speed. Vision Research, 50, 65-71. doi:10.1016/j.visres.2009.10.009

Norman, J. F., Clayton, A. M., Shular, C. F., \& Thompson, S. R. (2004). Aging and the perception of depth and 3-D shape from motion parallax. Psychology and Aging, 19, 506-514. doi:10.1037/0882-7974.19.3.506

Norman, J. F., Crabtree, C. E., Herrmann, M., Thompson, S. R., Shular, C. F., \& Clayton, A. M. (2006). Aging and the perception of 3-D shape from dynamic patterns of binocular disparity. Perception \& Psychophysics, 68, 94-101. doi:10.3758/BF03193659

Norman, J. F., Crabtree, C. E., Norman, H. F., Moncrief, B. K., Herrmann, M., \& Kapley, N. (2006). Aging and the visual, haptic, and cross-modal perception of natural object shape. Perception, 35, 1383-1395. doi:10.1068/p5504

Norman, J. F., Dawson, T. E., \& Butler, A. K. (2000). The effects of age upon the perception of depth and 3-D shape from differential motion and binocular disparity. Perception, 29, 1335-1359. doi:10.1068/p3111

Norman, J. F., \& Lappin, J. S. (1992). The detection of surface curvatures defined by optical motion. Perception \& Psychophysics, 51, 386-396. doi:10.3758/BF03211632 
Norman, J. F., Lappin, J. S., \& Zucker, S. W. (1991). The discriminability of smooth stereoscopic surfaces. Perception, 20, 789-807. doi: $10.1068 / \mathrm{p} 200789$

Norman, J. F., Norman, H. F., Craft, A. E., Walton, C. L., Bartholomew, A. N., Burton, C. L., ... Crabtree, C. E. (2008). Stereopsis and aging. Vision Research, 48, 2456-2465. doi:10.1016/j.visres.2008.08.008

Norman, J. F., Ross, H. E., Hawkes, L. M., \& Long, J. R. (2003). Aging and the perception of speed. Perception, 32, 85-96. doi: $10.1068 / \mathrm{p} 3478$

Norman, J. F., Swindle, J. M., Jennings, L. R., Mullins, E. M., \& Beers, A. M. (2009). Stereoscopic shape discrimination is well preserved across changes in object size. Acta Psychologica, 131, 129-135. doi:10.1016/j.actpsy.2009.03.009

Ogle, K. N. (1950). Researches in binocular vision. Philadelphia, PA: Saunders.

Ogle, K. N. (1958). Present status of our knowledge of stereoscopic vision. A. M. A. Archives of Ophthalmology, 60, 755-774.

Patterson, R., Cayko, R., Short, G. L., Flanagan, R., Moe, L., Taylor, E., \& Day, P. (1995). Temporal integration differences between crossed and uncrossed stereoscopic mechanisms. Perception \& Psychophysics, 57, 891-897.

Patterson, R., Moe, L., \& Hewitt, T. (1992). Factors that affect depth perception in stereoscopic displays. Human Factors, 34, 655-667.

Raghuram, A., Lakshminarayanan, V., \& Khanna, R. (2005). Psychophysical estimation of speed discrimination: II. Aging effects. Journal of the Optical Society of America. A, 22, 2269-2280.

Rogers, B., \& Graham, M. (1979). Motion parallax as an independent cue for depth perception. Perception, 8, 125-134.

Rogers, B., \& Graham, M. (1982). Similarities between motion parallax and stereopsis in human depth perception. Vision Research, 22, 261-270

Saidpour, A., Braunstein, M. L., \& Hoffman, D. D. (1994). Interpolation across surface discontinuities in structure from motion. Perception \& Psychophysics, 55, 611-622.

Snowden, R. J., \& Kavanagh, E. (2006). Motion perception in the ageing visual system: Minimum motion, motion coherence, and speed discrimination thresholds. Perception, 35, 9-24.

Sperling, G., Landy, M. S., Dosher, B. A., \& Perkins, M. E. (1989). Kinetic depth effect and identification of shape. Journal of Experimental Psychology. Human Perception and Performance, 15, 826-840.

Terzopoulos, D. (1988). The computation of visible-surface representations. IEEE Transactions on Pattern Analysis and Machine Intelligence, 10, 417-438.
Todd, J. T., Akerstrom, R. A., Reichel, F. D., \& Hayes, W. (1988). Apparent rotation in three-dimensional space: Effects of temporal, spatial, and structural factors. Perception \& Psychophysics, 43, $179-188$.

Todd, J. T., \& Norman, J. F. (1991). The visual perception of smoothly curved surfaces from minimal apparent motion sequences. Perception \& Psychophysics, 50, 509-523. doi:10.3758/BF03207535

Ullman, S. (1979). The interpretation of structure from motion. Proceedings of the Royal Society B, 203, 405-426.

Uttal, W. R. (1985). The detection of nonplanar surfaces in visual space. Hillsdale, NJ: Erlbaum.

Uttal, W. R., Davis, N. S., Welke, C., \& Kakarala, R. (1988). The reconstruction of static visual forms from sparse dotted samples. Perception \& Psychophysics, 43, 223-240.

Van Damme, W. J. M., \& Van de Grind, W. A. (1993). Active vision and the identification of three-dimensional shape. Vision Research, 33, 1581-1587.

von Helmholtz, H. (1925). Treatise on physiological optics (Vol. 3). Rochester, NY: Optical Society of America. Original work published 1867.

Vreven, D. (2006). 3D shape discrimination using relative disparity derivatives. Vision Research, 46, 4181-4192.

Vuong, Q. C., Domini, F., \& Caudek, C. (2004). Evidence for patchwork approximation of shape primitives. Perception \& Psychophysics, 66, 1246-1259.

Vuong, Q. C., Domini, F., \& Caudek, C. (2006). Disparity and shading cues cooperate for surface interpolation. Perception, 35, 145-155.

Wallach, H., \& O'Connell, D. N. (1953). The kinetic depth effect. Journal of Experimental Psychology, 45, 205-217.

Wheatstone, C. (1838). Contributions to the physiology of vision: Part the first. On some remarkable, and hitherto unobserved, phenomena of binocular vision. Philosophical Transactions of the Royal Society, 128, 371-394.

Wilcox, L. M., \& Duke, P. A. (2005). Spatial and temporal properties of stereoscopic surface interpolation. Perception, 34, 1325-1338.

Yang, Y., \& Blake, R. (1995). On the accuracy of surface reconstruction from disparity interpolation. Vision Research, 35, 949-960.

Yang, Y., Liang, Z., Li, G., Wang, Y., \& Zhou, Y. (2009). Aging affects response variability of $\mathrm{V} 1$ and MT neurons in rhesus monkeys. Brain Research, 1274, 21-27.

Yang, Y., Zhang, J., Liang, Z., Li, G., Wang, Y., Ma, Y., ... Leventhal, A. G. (2009). Aging affects the neural representation of speed in macaque area MT. Cerebral Cortex, 19, 1957-1967. 